\title{
Centralized scheduling, routing tree in WiMAX mesh networks
}

\begin{abstract}
In the last few years, demand for high speed internet access service has increased greatly so the IEEE 802.16 working group on broadband wireless access (BWA) is developing the worldwide interoperability for microwave access (WiMAX) standard for wireless metropolitan area networks (MANs) which aims to provide broadband wireless last mile access, easy deployment, and high speed data rate for large spanning area. This paper propose an efficient routing and collision free centralized scheduling (CS) algorithms using single channel single transceiver system in WiMAX mesh network, which introduces the cross layer concept between the network layer and media access controller (MAC) layer. The proposed approach has improved the system performance in the aspects of scheduling length, channel utilization ratio (CUR), and the throughput of the system.
\end{abstract}

Keyword: WiMAX; Routing trees; Cross layer; Mesh network; Centralized scheduling; CS algorithms 